\title{
MicroRNA-1 affects the development of the neural crest and craniofacial skeleton via the mitochondrial apoptosis pathway
}

\author{
NA ZHAO $^{1 *}$, WENHAO QIN ${ }^{1 *}$, DONGYUE WANG ${ }^{1}$, ANAKARINA GONZÁLEZ RAQUEL $^{1}$, \\ LICHAN YUAN $^{1}$, YELIN MAO $^{2}$, CHANGYAN MA $^{3}$, ZHONGDANG XIAO $^{4}$ and JUNQING MA ${ }^{1}$ \\ ${ }^{1}$ Jiangsu Key Laboratory of Oral Diseases, Nanjing Medical University, Nanjing, Jiangsu 210029; \\ ${ }^{2}$ Department of Orthodontics, The Affiliated Stomatology Hospital of Suzhou Vocational Health College, Suzhou, \\ Jiangsu 215002; ${ }^{3}$ Department of Medical Genetics, Nanjing Medical University, Nanjing, Jiangsu 211166; \\ ${ }^{4}$ State Key Laboratory of Bioelectronics, Southeast University, Nanjing, Jiangsu 210096, P.R. China
}

Received October 29, 2019; Accepted July 17, 2020

DOI: $10.3892 /$ etm.2021.9810

\begin{abstract}
The neural crest is one of the key features of craniofacial development. MicroRNA-1 (miR-1) is a single-stranded noncoding RNA that serves an important role in embryonic development. However, the function of miR-1 in neural crest cells (NCCs) is unknown. Therefore, to evaluate the role of miR-1 in NCC development, a miR-1 mutant zebrafish was generated in the current study. Mouse NCCs were isolated from the first branchial arch of embryos at gestational day E9.5, and miR-1 was silenced using a miR-1 inhibitor. To the best of our knowledge, the present study was the first to report that homozygous zebrafish lacking miR-1 exhibited developmental defects in NCC-derived craniofacial bones, heart, melanocytes and iridophores. These defects may be caused by an increase in apoptosis of NCCs during their migration and differentiation in embryonic development. Moreover, the apoptosis analysis and western blotting results demonstrated that this effect was modulated via the mitochondrial apoptosis pathway, and miR-1 inhibited NCC apoptosis by modulating this pathway. These results collectively suggested that miR-1 in NCCs may be essential for craniofacial development.
\end{abstract}

\section{Introduction}

The neural crest (NC) is a multipotent cell population and is one of the key features of craniofacial development (1). Neural crest cells (NCCs) originate from the dorsal neural tube and generate numerous cell types, including cartilage and pigment

Correspondence to: Professor Junqing Ma, Jiangsu Key Laboratory of Oral Diseases, Nanjing Medical University, 136 Hanzhong Road, Nanjing, Jiangsu 210029, P.R. China

E-mail: jma@njmu.edu.cn

*Contributed equally

Key words: microRNA-1, mitochondrial apoptosis pathway, neural crest, apoptosis, zebrafish cells (2). Defective development of NC has been demonstrated to result in a number of syndromes, such as Treacher Collins and DiGeorge syndrome, which are characterized by developmental defects in the craniofacial region (3-5). In humans, craniofacial malformations have been reported to be associated with $75 \%$ of all congenital birth malformations (6) and are accompanied by abnormal brain development and poor prognosis.

MicroRNAs (miRNAs/miRs) have been demonstrated to regulate $\sim 30 \%$ of genes in Caenorhabditis elegans, mouse and human and serve an important role in embryonic development (7). The Homo sapiens (hsa)-miR-1 family includes two miRNAs, hsa-miR-1-1 and hsa-miR-1-2, which exhibit the same mature sequence, although they are encoded by different genes (8). Previous studies have indicated that miR-1 participates in regulating the development of the heart and skeletal muscle $(9,10)$. The outflow track of the heart, as well as the tendons, muscles and craniofacial structures, have all been reported to be derivatives of the NC (1). miR-1 has been revealed to be conserved and important for embryonic development in zebrafish, especially NC development (11). However, the specific role of miR-1 in NCCs is currently unknown.

Zebrafish exhibit rapid embryonic development, a short life cycle and large spawning capacity. In addition, the embryos are transparent, so the process of embryonic skeletal development can be observed directly (12). Therefore, zebrafish are a suitable animal model to study the function of genes in craniofacial development. In the present study, the miR-1-knockout zebrafish model was a homozygous mutant, which was used to examine the role of miR-1 in NCCs.

The current study aimed to evaluate the role of miR-1 in the development of $\mathrm{NC}$ using a miR-1 deficient zebrafish model.

\section{Materials and methods}

Animals. The wild-type (WT) zebrafish embryos were of the Tuebingen strain. WT and homozygous miR-1 mutant zebrafish ( $\mathrm{n}=60$ per group) were purchased from the Institute of Model Animals of Nanjing University. The primers used for genotyping of the miR-1 mutant zebrafish, the sequence of the mutant allele and the identification of the genotype are 
presented in Tables SI and SII and Fig. S1. The embryos were grown in Holtfreter's solution $\left(0.05 \mathrm{~g} / 1 \mathrm{KCL}, 0.1 \mathrm{~g} / 1 \mathrm{CaCl}_{2}\right.$, $0.2 \mathrm{~g} / 1 \mathrm{NaHCO}_{3}$ and $\left.3.5 \mathrm{~g} / \mathrm{l} \mathrm{Nacl}\right)$ with a $14 \mathrm{~h} \mathrm{light} / 10 \mathrm{~h}$ dark cycle at $28.5^{\circ} \mathrm{C}$ in the zebrafish facility of the Model Animal Research Center, Nanjing University (Nanjing, China). The zebrafish were fed three times a day with pellet food, and their health and behavior was monitored daily. All experiments were approved by the Ethics Committee of the School of Stomatology of Nanjing Medical University, and all procedures were performed according to the guidelines of the Animal Care Committee of Nanjing Medical University.

Whole mount in situ hybridization. Whole mount in situ hybridization was performed using standard procedures with slight modifications as previously described (13). Probes were synthesized using a DIG RNA labeling kit (Roche Diagnostics). The probes of forkhead box D3 (foxd3), muscle segment homeobox-b (msxb), snail homolog $1 \mathrm{~b}$ (snailb), transcription factor AP2a (tfap2a), distal-less $3(d l x 3 b)$, neurog1 (ngnl) (Table SIII) were designed using Primer 5.0 software (Premier Biotech, Inc.). Embryos ( $\mathrm{n}=5$ per group) at $24 \mathrm{~h}$ post-fertilization (hpf) were collected, fixed in $4 \%$ paraformaldehyde overnight at $4^{\circ} \mathrm{C}$ and subjected to gradient dehydration with methanol $(25,50,75$ and $100 \%)$. The embryos were maintained in $100 \%$ methanol at $-20^{\circ} \mathrm{C}$ for subsequent use. Following rehydration at gradient dilutions using methanol/PBS, the embryos were treated with proteinase K $(10 \mu \mathrm{g} / \mathrm{ml}$; Gibco; Thermo Fisher Scientific, Inc.) at room temperature for $10 \mathrm{~min}$. Subsequently, embryos were prehybridized with the hybridization solution at $65^{\circ} \mathrm{C}$ for $2 \mathrm{~h}$. In situ hybridization signals were detected by sheep anti-digoxigenin-AP Fab-fragments (Roche Diagnostics) and the color reaction was carried out by staining with BCIP/NBT (Roche Diagnostics) as previously described (14).

Alcian blue staining. Zebrafish embryos at $96 \mathrm{hpf}(\mathrm{n}=5$ per group) were fixed in $4 \%$ paraformaldehyde overnight at $4^{\circ} \mathrm{C}$, dehydrated with ethanol (50 and 100\%) and stained with Alcian blue $(0.15 \mathrm{mg} / \mathrm{ml}$; Sigma-Aldrich; Merck KGaA) overnight at room temperature (15). After the embryonic cartilage was stained, the embryos were incubated in saturated boron overnight at room temperature. Subsequently, bleaching was performed in a mixture of $3 \% \mathrm{H}_{2} \mathrm{O}_{2}$ and $1 \% \mathrm{KOH}$ at a ratio of $15: 85 \mathrm{v} / \mathrm{v}$, and the specimens were digested in $1 \%$ trypsin at $37^{\circ} \mathrm{C}$ until all embryonic tissues were degraded. The digestion mixture was replaced with $1 \% \mathrm{KOH}$ solution, and subsequently the specimens were incubated in a glycerol gradient $(25,50,75$ and $100 \%)$. The embryos were observed using a stereomicroscope (magnification, x20).

Histology and immunostaining. For whole mount immunostaining, the embryos ( $n=5$ per group) were fixed in $4 \%$ paraformaldehyde overnight at $4{ }^{\circ} \mathrm{C}$ pretreated with proteinase $\mathrm{K}(10 \mu \mathrm{g} / \mathrm{ml})$ at $37^{\circ} \mathrm{C}$ for $30 \mathrm{~min}$ and blocked in 2\% BSA solution (BioFroxx) at room temperature for $1 \mathrm{~h}$. Subsequently, the embryos were stained with an anti-phospho-histone H3 (PHH3; 1:100; cat. no. 3377; Cell Signaling Technology, Inc.) antibody overnight at $4^{\circ} \mathrm{C}$ and then with an anti-rabbit Alexa Fluor@594 (1:400; Jackson ImmunoResearch Laboratories, Inc.) at $4^{\circ} \mathrm{C}$ for $2 \mathrm{~h}$. A TUNEL assay was performed using an In Situ Cell Death Detection kit (Roche Diagnostics) as previously described (16). The staining was subsequently observed using laser scanning confocal microscopy (magnification, x20) (17).

Culture of NCCs. Mouse embryos were harvested at gestational day E9.5 according to a previously published protocol (18). The pregnant adult mice $(n=3$; Model Animal Research Center, Yangzhou University) were sacrificed by cervical dislocation following anesthesia with sodium pentobarbital $(50 \mathrm{mg} / \mathrm{kg})$. The first branchial arch of the embryos was separated, washed in PBS and dissociated in $0.25 \%$ trypsin-EDTA (Gibco; Thermo Fisher Scientific, Inc.) for $30 \mathrm{~min}$ at $37^{\circ} \mathrm{C}$. The separated cells were plated into a dish in Nutrient Mixture F-12 (1:1)media (Gibco; Thermo Fisher Scientific, Inc.) with $15 \%$ FBS (ScienCell Research Laboratories, Inc.), supplemented with $100 \mathrm{U} / \mathrm{ml}$ penicillin-streptomycin (PS), $100 \mathrm{mg} / \mathrm{ml} \mathrm{L-glutamate,} 0.1 \mathrm{mM}$ minimum essential medium non-essential amino acids (Gibco; Thermo Fisher Scientific, Inc.) and 1,000 U/ml leukemia inhibitory factor (PeproTech, Inc.). The inhibitor (5'-AUA CAUACUUCUUUACAUUCCA-3'; $100 \mathrm{nM}$ ) and mimics (forward, 5'-UGGAAUGUAAAGAAGUAUGUAU-3'; and reverse, 5'-AUACAUACUUCUUUACAUUCCA-3'; $50 \mathrm{nM}$ ) of miR-1 and their negative controls (NCs; inhibitor NC, 5'-CAG UACUUUUGUGUAGUACAAA-3'; mimics NC, 5'-UUU GUACUACACAAAAGUACUG-3'; 5'-CAGUACUUUUG UGUAGUACAAA-3'; all Guangzhou RiboBio Co., Ltd.) were transfected into NCCs when cells grew to $50 \%$ density using a riboFECT ${ }^{\mathrm{TM}}$ CP Transfection kit (Guangzhou RiboBio Co., Ltd.) according to the manufacturer's protocol.

Cell Counting Kit-8 (CCK-8) assay. CCK-8 assay (Dojindo Molecular Technologies, Inc.) was used to evaluate cell proliferation according to the manufacturer's protocol. NCCs were seeded at $2 \times 10^{3}$ cells/well in 96 -well plates. The cells were incubated at $37^{\circ} \mathrm{C}$ for $12 \mathrm{~h}$ (day 0) and transfected with the miR-1 inhibitor. CCK-8 reagent in Nutrient Mixture F-12 media was added to each well at the specified time points (days $0,1,2,3$ and 4). Following 1-h incubation at $37^{\circ} \mathrm{C}$, the optical density was measured at $450 \mathrm{~nm}$ using a microplate reader with three blank controls.

Wound healing assay. NCCs were seeded at $1.5 \times 10^{5}$ cells/well in a 6 -well plate. Following transfection with the miR-1 inhibitor, a wound was created with a $10 \mu \mathrm{l}$ pipette tip, and the cells were incubated in serum-free medium at $37^{\circ} \mathrm{C}$ for $24 \mathrm{~h}$. Cell migration was observed at 0,12 and $24 \mathrm{~h}$ using a Leica DMIRE2 microscope (magnification, x100; Leica Microsystems $\mathrm{GmbH}$ ) in phase contrast mode (19).

Cell cycle and apoptosis analysis. NCCs transfected with the miR-1 inhibitor were cultured for $24 \mathrm{~h}$ without PS. Following an additional 48-h culture, cell cycle and apoptosis analyses were performed as previously described (20) using flow cytometry (FACSCalibur; BD Biosciences). For cell cycle analysis, the cells $\left(\sim 1 \times 10^{4}\right)$ were fixed overnight with $75 \%$ ethanol at $4^{\circ} \mathrm{C}$ in the dark, and then stained with propidium iodide (PI; MultiSciences Biotech Co., Ltd.) containing RNase. Apoptosis was detected by harvesting cells using a centrifuge 
A

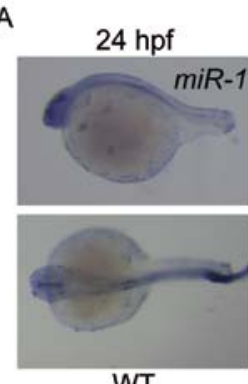

WT

D
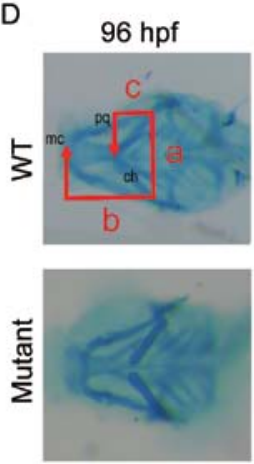

B

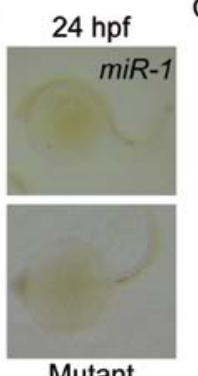

E

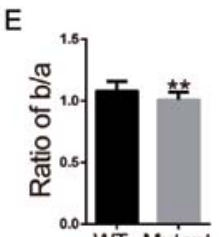

\section{F}

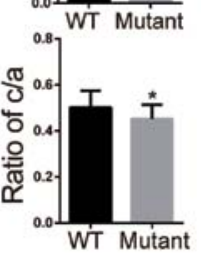

C
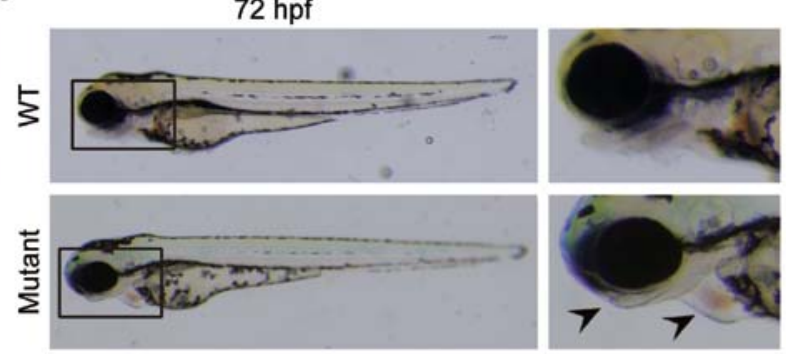

G

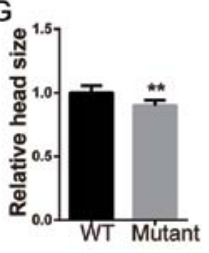

$\mathrm{H}$
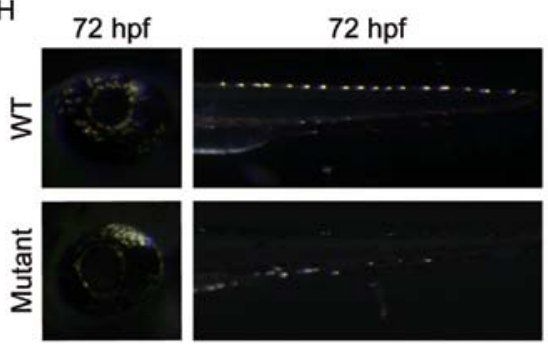

Figure 1. Loss of miR-1 in zebrafish results in craniofacial, pigment cell and cardiac defects. In situ hybridization of miR-1 in (A) wild-type and (B) mutant zebrafish at $24 \mathrm{hpf}$. (C) Zebrafish larvae at $72 \mathrm{hpf}$ with mutants exhibiting mandibular retrognathia (left arrow) and edema around the heart (right arrow). (D) Alcian blue staining at 96 hpf. (E) Quantitative analysis of b/a. (F) Quantitative analysis of c/a. a, width between ch; b, distance between mc and ch; and c, the distance between pq and ch. (G) Quantitative analysis of head size. (H) Distribution and number of iridophores at 72 hpf. Data are presented as the mean \pm standard deviation $(\mathrm{n}=5)$. ${ }^{*} \mathrm{P}<0.05$ and ${ }^{* *} \mathrm{P}<0.01$ vs. WT. WT, wild-type; hpf, hours post-fertilization; miR-1, microRNA-1; mc, Meckel's cartilage; pq, palatoquadrate; ch, ceratohyal cartilage.

at room temperature $(5 \mathrm{~min}, 210 \mathrm{xg})$ and staining the cells $\left(\sim 1 \times 10^{4}\right)$ with an Annexin V-FITC Apoptosis Detection kit (BD Pharmingen; BD Biosciences).

$\mathrm{PI}^{-} /$Annexin V-FITC ${ }^{+}$cells (early apoptotic population) and $\mathrm{PI}^{+} /$Annexin $\mathrm{V}^{-F I T C}{ }^{+}$cells (late apoptotic population) were sorted. Analysis was performed using FACScan cytometer (BD Biosciences) and FlowJo V7 software (Tree Star).

Apoptotic cell staining. NCCs were seeded at $6 \times 10^{4}$ cells/well in a 24-well plate, transfected with the miR-1 inhibitor and then cultured for 3 days at $37^{\circ} \mathrm{C}$. Acridine orange/ethidium bromide $(\mathrm{AO} / \mathrm{EB})$ staining was performed using an $\mathrm{AO} / \mathrm{EB}$ Detection kit (Biotechgate) according to the manufacturer's instructions. After fixing in $4 \%$ paraformaldehyde at $4{ }^{\circ} \mathrm{C}$ for $15 \mathrm{~min}$, TUNEL staining of NCCs was performed using an In Situ Cell Death Detection kit (Roche Diagnostics) according to the manufacturer's instructions. The cells were observed using fluorescence microscopy (magnification, x400; Leica Microsystems, Inc.).

Reverse transcription-quantitative PCR (RT-qPCR). Total NCCs RNA was isolated using TRIzol ${ }^{\circledR}$ reagent (Invitrogen; Thermo Fisher Scientific, Inc.), as previously described (21). The miRNA expression levels of miR-1 relative to the internal control U6 were evaluated after reverse transcription $\left(37^{\circ} \mathrm{C}\right.$ for $60 \mathrm{~min}$ and $85^{\circ} \mathrm{C}$ for $5 \mathrm{~min}$ ) using an All-in-One ${ }^{\mathrm{TM}}$ miRNA RT-qPCR Detection kit (GeneCopoeia, Inc.), the thermocycling conditions were set according to the manufacturer's instructions. The miR-1 (5'-CGCGTGGAATGTAAAGAA GTATGTA-3') and U6 (5'-AAAACAGCAATATGGAGC
GC-3') forward primers and universal reverse primers (cat. no. QP015) were purchased from GeneCopoeia, Inc. Relative expression levels were calculated using the $2^{-\Delta \Delta \mathrm{Cq}}$ method (22).

Western blot analysis. Proteins of cells were extracted using RIPA buffer (Beyotime Institute of Biotechnology), boiled for $5 \mathrm{~min}$ and analyzed its protein concentrations using a Bicinchoninic Acid Protein Assay Kit (Beyotime Institute of Biotechnology). The proteins were separated using $10 \%$ or $12 \%$ SDS-PAGE as previously described (19), transferred onto PVDF membranes and then blocked with 5\% BSA at room temperature for $2 \mathrm{~h}$. Primary antibodies against BCL-2, poly ADP-ribose polymerase (PARP), pro- and cleaved-caspase-9 (1:500; cat. nos. 12789, 13371 and 10380, respectively; Wuhan Sanying Biotechnology), pro- and cleaved-caspase-3 (1:1,000; cat. no. 9661S; Cell Signaling Technology, Inc.) and $\beta$-actin (1:1,000; cat. no. sc-47778; Santa Cruz Biotechnology, Inc.) were used overnight at $4^{\circ} \mathrm{C}$. Next, the membranes were incubated with HRP-labeled Goat Anti-Rabbit or HRP-labeled Goat Anti-mouse secondary antibody (1:8,000, cat. nos. 474-1506 and 474-1806, respectively; SeraCare KPL) at room temperature for $1 \mathrm{~h}$ and visualized by enhanced chemiluminescence (Thermo Fisher Scientific, Inc.). Semi-quantitative analysis was performed using Image J 1.52 software (National Institutes of Health).

Statistical analysis. All data are presented as the mean \pm standard deviation. Differences between two groups were analyzed using GraphPad Prism v6 statistical software (GraphPad Software Inc.). Unpaired Student's t-test was used for the 
A

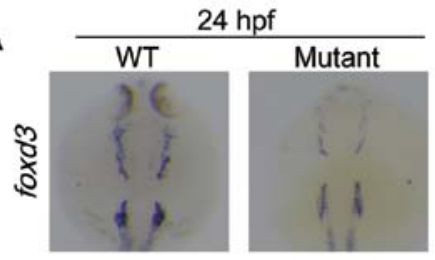

D

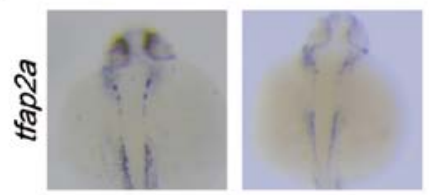

G

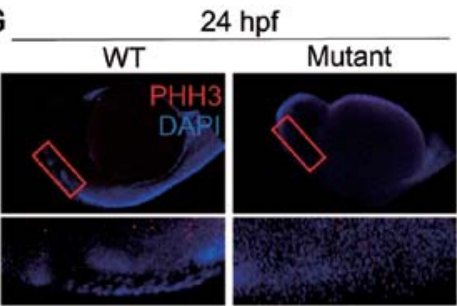

B

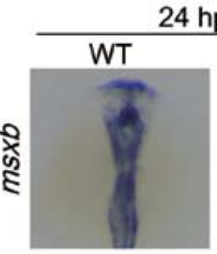

E

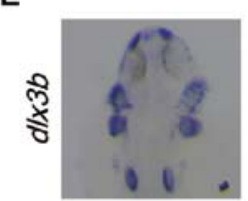

H

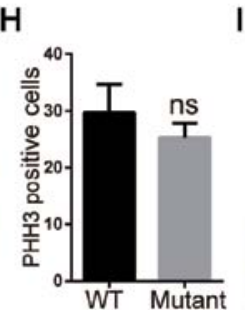

I
$24 \mathrm{hpf}$
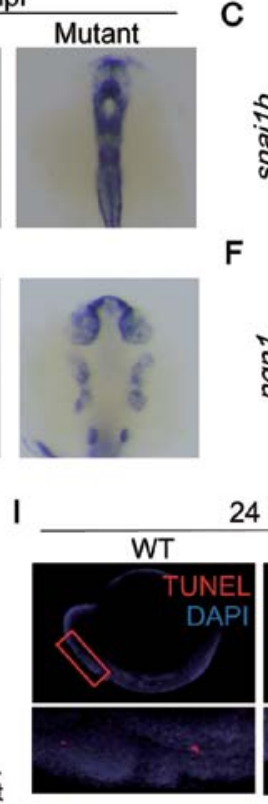

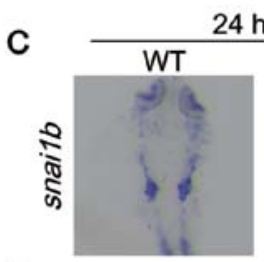

$\mathbf{F}$

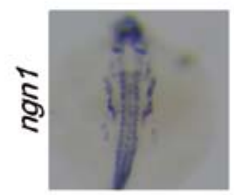

$24 \mathrm{hpf}$

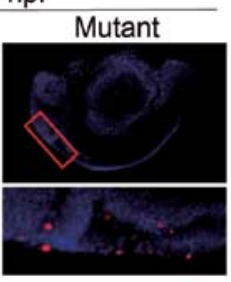

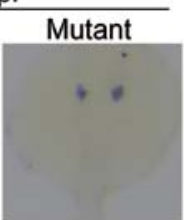

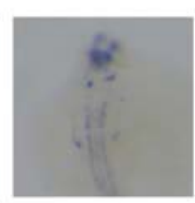

$\mathrm{J}$

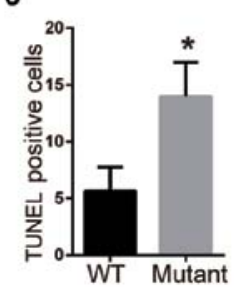

Figure 2. MicroRNA-1 knockout affects neural crest cell apoptosis during the migration and differentiation periods of embryonic development. In situ hybridization of zebrafish at $24 \mathrm{hpf}$ with (A) foxd3, (B) msxb, (C) snailb, (D) tfap2a, (E) dlx3b and (F) ngn1 probes. (G) Whole mount immunostaining of zebrafish at $24 \mathrm{hpf}$ with PHH3 (red) and DAPI (blue). (H) Quantitative analysis of immunostaining. (I) TUNEL staining at 24 hpf with TUNEL (red) and DAPI (blue). (J) Quantitative analysis of TUNEL staining. Data are presented as the mean \pm standard deviation (n=5). *P<0.05 vs. WT. WT, wild-type; foxd3, forkhead box D3; msxb, muscle segment homeobox-B; snailb, snail homolog 1b; tfap2a, transcription factor AP2A; dlx3b, distal-less 3; ngn1, neurog1; PHH3, phospho-histone H3; hpf, hours post fertilization; ns, not significant.

statistical analysis. $\mathrm{P}<0.05$ was considered to indicate a statistically significant difference.

\section{Results}

Loss of miR-1 in zebrafish results in craniofacial, pigment cell and cardiac defects. In the present study, a miR-1 mutant zebrafish model was generated to evaluate the role of miR-1 in the development of NC. The expression of miR-1 was primarily localized in the head, pharyngeal arch and pectoral fin of zebrafish (Fig. 1A). In the miR-1 mutant zebrafish, the expression of miR-1 was suppressed in the aforementioned areas (Fig. 1B).

A number of abnormal phenotypes were observed in the mutant compared with the WT zebrafish, including a smaller head, a decreased mandibular arch length and mandibular retrognathia (Fig. 1C-G). In the mutant zebrafish, the number of melanocytes and iridophores (NCC-derived pigment cells) was decreased (Fig. 1C and $\mathrm{H}$ ). In addition, the mutant zebrafish exhibited abnormal cardiac development with enlarged hearts compared with those of the WT zebrafish (Fig. 1C). A quantitative analysis of the association among the palatal square joint, McGuire cartilage and hyoid small horn cartilage has been previously performed (23). In the present study, the head size and mandibular arch length were decreased in the mutant group compared with those in the WT group (Fig. 1D-G).

These results suggested that the lack of miR-1 affected the development of NCCs in zebrafish, which resulted in severe phenotypic defects.

miR-1 affects NCC apoptosis during the migration and differentiation periods in embryonic development. To determine which stage of NC development was affected by miR-1, the expression of marker genes of specific stages (foxd3, msxb, snailb, tfap $2 a, d l x 3 b$ and $n g n 1)$ was detected via in situ hybridization. These genes have been demonstrated to be associated with the induction, specialization, migration and differentiation of NCCs $(24,25)$.

Foxd3 has been indicated to serve an important role in NC derived tissues, such as the pharyngeal arch (24). In the present study, two regions of NCCs were labelled with the foxd3 probe in WT and mutant zebrafish; however, the mutant group appeared to exhibit lower expression levels of foxd 3 compared with that in the WT group (Fig. 2A). This indicated that the migration of NCCs may be suppressed in the miR-1 mutant group. Msxb in zebrafish has also been reported to be required for NCC survival (25). However, $m s x b$ expression exhibited no difference between the two groups (Fig. 2B). Therefore, it was speculated that the lack of miR-1 affected the migration, but not the survival of NCCs.

To examine migration, the expression of snailb and tfap $2 a$ was also examined in the NC area of the head. In situ hybridization indicated that snailb and tfap $2 a$ expression appeared to be decreased in the mutant group at $24 \mathrm{hpf}$ compared with that in the WT group (Fig. 2C and D). These results suggested that migration was inhibited in the mutant zebrafish.

During embryonic development in zebrafish, the first and second pharyngeal arch develop into the mandible and hyoid arches (1). The results of the present study indicated that the expression of $d l x 3 b$ in miR-1 mutant embryos appeared to be decreased compared with that in the WT group (Fig. 2E). This was consistent with the mutant zebrafish phenotype, namely lower mandibular arch length and mandibular retrognathia, which was observed in the current study. Knockdown of miR-1 
A

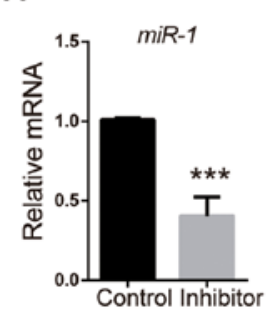

E

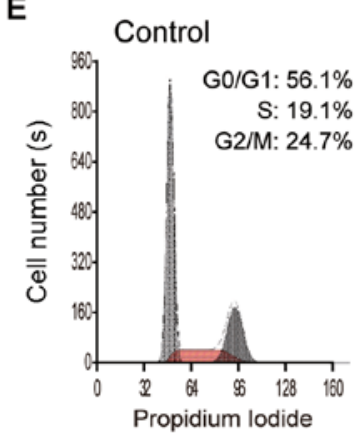

$\circ$

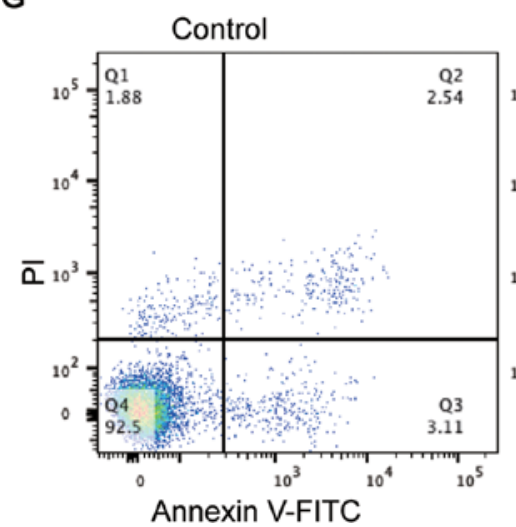

B

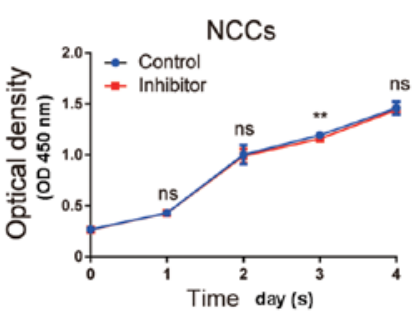

Inhibitor

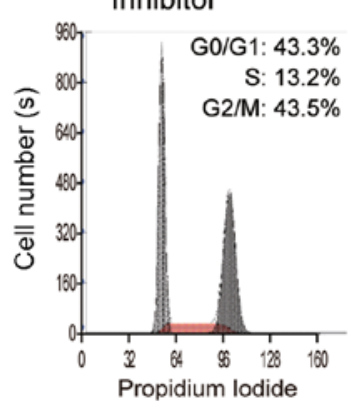

Inhibitor
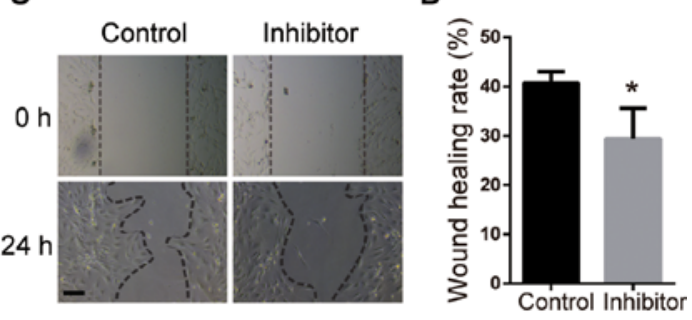

$\mathrm{F}$
D

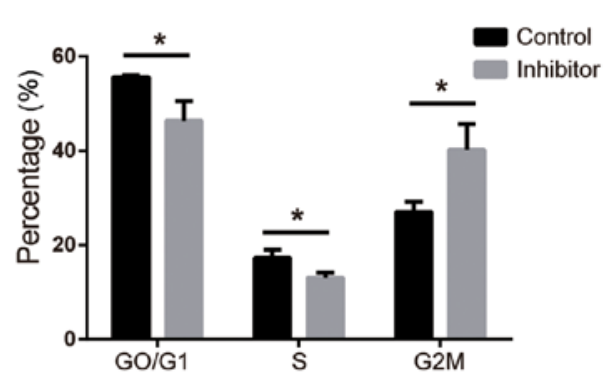

H
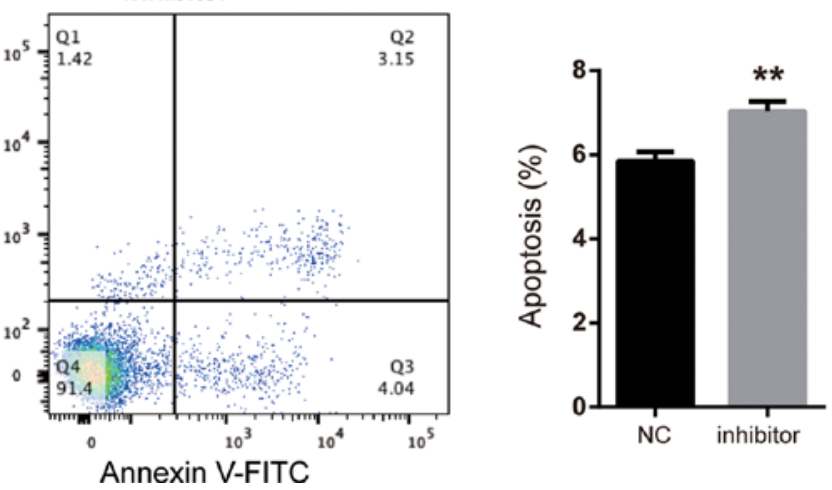

Figure 3. miR-1 regulates the function of NCCs in vitro. (A) miR-1 expression assessed via reverse transcription-quantitative PCR following transfection with the miR-1 inhibitor. (B) Cell proliferation of NCCs analyzed by Cell Counting Kit-8 assay. (C) Wound healing assay of NCCs. Scale bar, $100 \mu \mathrm{m}$. (D) Quantitative analysis of wound healing assay. (E) Cell cycle analysis of NCCs performed using flow cytometry. (F) Quantitative analysis of cell cycle analysis. (G) Apoptosis analysis of NCCs evaluated via flow cytometry. (H) Quantitative analysis of apoptosis analysis. Data are presented as the mean \pm standard deviation ( $\mathrm{n}=3) .{ }^{*} \mathrm{P}<0.05,{ }^{* *} \mathrm{P}<0.01$ and ${ }^{* * *} \mathrm{P}<0.001$ vs. Control. NCCs, neural crest cells; miR-1, microRNA-1; PI, propidium iodide.

appeared to decrease in $n g n l$ expression compared with that in the WT group (Fig. 2F). This evidence collectively suggested that miR-1 may serve an important role during the migration and differentiation periods of NCCs.

Cell proliferation, which was assessed by immunofluorescence staining of $\mathrm{PHH} 3$, exhibited no differences between the two groups (Fig. 2G and H). An assessment of apoptosis in zebrafish embryos at $24 \mathrm{hpf}$ was performed using a whole-mount TUNEL assay. The results demonstrated that miR-1-knockout embryos exhibited an increased level apoptosis compared with that in the WT embryos (Fig. 2I and J). These results demonstrated the importance of miR-1 in regulating apoptosis during $\mathrm{NC}$ development.

miR-1 regulates NCC functions in vitro. To examine the function of miR-1 in vitro, miR-1 was knocked down in NCCs isolated from mice using a miR-1 inhibitor (Fig. 3A). Following inhibition of miR-1, the proliferative ability of NCCs was slightly reduced compared with that of the control group at day 3 , whereas no significant differences were observed at day 1, 2 and 4 (Fig. 3B). In addition, a wound healing assay indicated that the migratory rate of the miR-1 inhibitor group was decreased compared with the control group (Fig. 3C and D).

Subsequently, cell cycle and apoptosis analysis was performed via flow cytometry. The results of the cell cycle analysis suggested that knockdown of miR-1 increased the number of cells at the $\mathrm{G}_{2} / \mathrm{M}$ phase compared with that in the control group, indicating that cell cycle was arrested at $\mathrm{G}_{2} / \mathrm{M}$ phase. By contrast, the numbers of NCCs at the $G_{0} / G_{1}$ and $S$ phases were reduced compared with those in the control group (Fig. 3E and F). In addition, following transfection with the miR-1 inhibitor, the number of early and late apoptotic cells was increased compared with that in the control group (Fig. $3 \mathrm{G}$ and $\mathrm{H}$ ). These in vitro results demonstrated that miR-1 may be crucial for apoptosis in NCCs and were consistent with the in vivo observations.

miR-1 affects NCC apoptosis via the mitochondrial apoptosis pathway and caspase-3. Early and late apoptosis in mouse NCCs was detected via $\mathrm{AO} / \mathrm{EB}$ staining. The results 
A

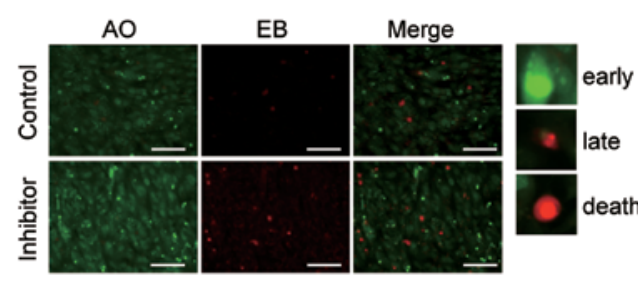

C

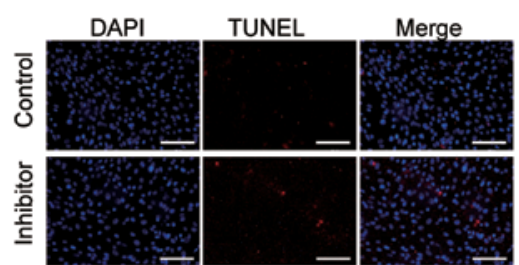

E

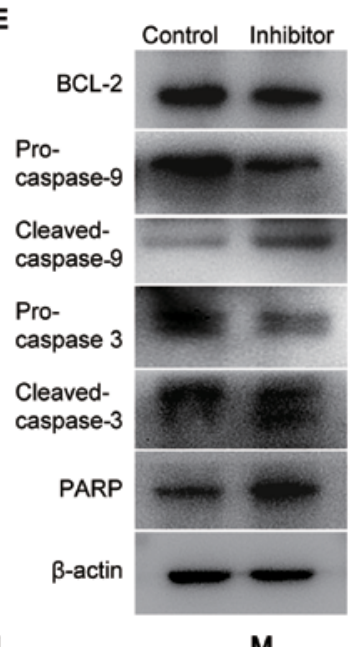

L

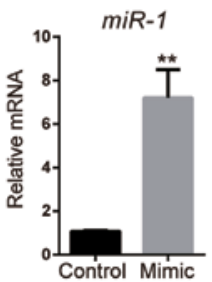

$F$
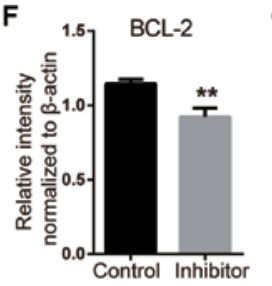

I

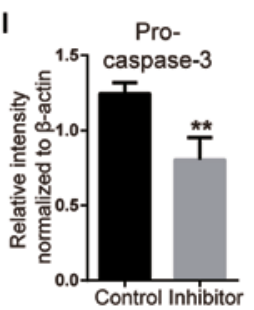

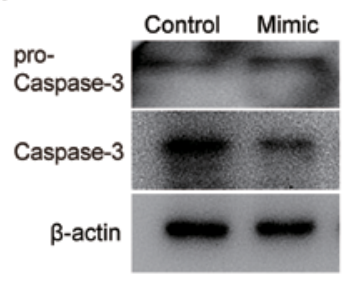

N
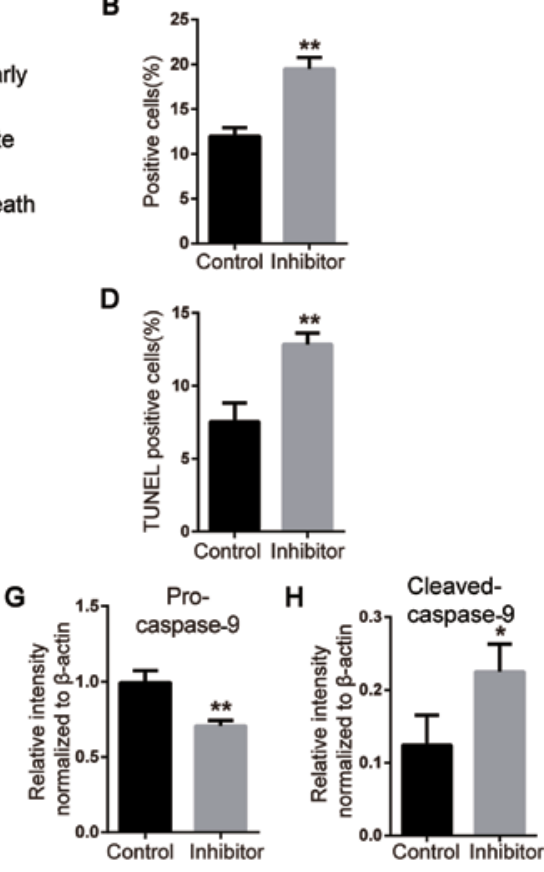

D
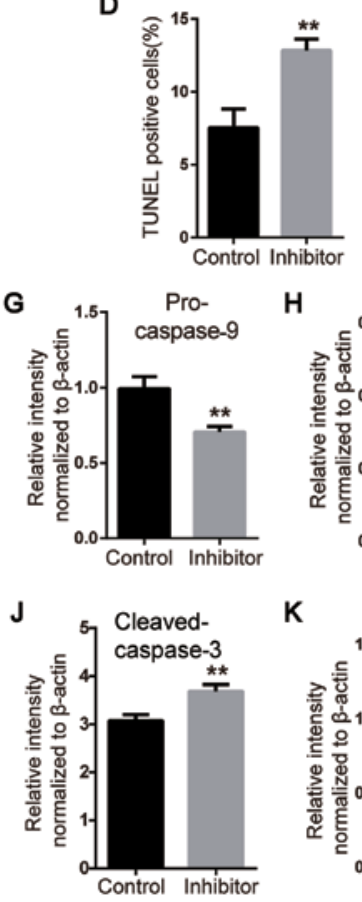

K
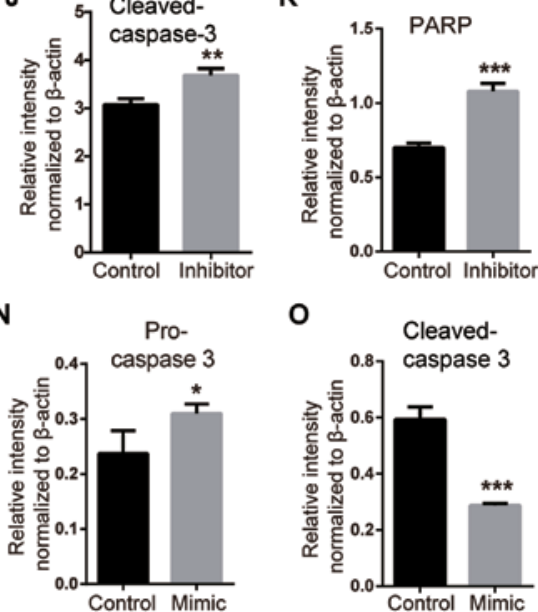

Figure 4. miR-1 affects the apoptosis of neural crest cells via the mitochondrial apoptosis pathway and caspase-3. (A) Detection of apoptosis on day 3 following miR-1 inhibitor transfection using AO/EB staining. Scale bar, $100 \mu \mathrm{m}$. (B) Quantitative analysis of AO/EB staining. (C) TUNEL staining of NCCs. Scale bar, $100 \mu \mathrm{m}$. (D) Quantitative analysis of TUNEL staining. (E) Protein levels of the mitochondrial apoptosis pathway markers BCL-2, pro- and cleaved-caspase-3, pro- and cleaved-caspase-9 and PARP detected via western blotting. (F-K) Quantification of the protein levels of (F) BCL-2, (G) pro-caspase-9, (H) cleaved-caspase-9, (I) pro-caspase-3, (J) cleaved-caspase-3 and (K) PARP, which are presented in (E). (L) miR-1 expression assessed by reverse transcription-quantitative PCR. (M) Expression levels of pro- and cleaved-caspase-3 after transfection with the miR-1 mimic. (N and O) Quantification of the protein levels. Data are presented as the mean \pm standard deviation $(\mathrm{n}=3)$. ${ }^{*} \mathrm{P}<0.05,{ }^{* *} \mathrm{P}<0.01$ and ${ }^{* * * *} \mathrm{P}<0.001$ vs. Control. miR-1, microR $\mathrm{NA}-1$; PARP, poly ADP-ribose polymerase, AO, acridine orange; EB, ethidium bromide.

demonstrated that the number of early and late apoptotic cells was increased in the miR-1 inhibitor group compared with that in the control group (Fig. 4A and B). In addition, TUNEL staining demonstrated that compared with the control group, an increased number of apoptotic cells was observed three days after transfection with miR-1 inhibitor (Fig. 4C and D).

Alterations in the apoptotic pathway were examined in mouse NCCs by detecting the expression levels of apoptosis-associated proteins using western blotting (Fig. 4E-K). The expression levels of cleaved-caspase-3 and -9 were increased in the miR-1 inhibitor group compared with those in the control group (Fig. $4 \mathrm{H}$ and $\mathrm{J}$ ), whereas the expression levels of pro-caspase-3 were decreased in the miR-1 inhibitor group compared with those in the control group (Fig. 4I). The expression levels of BCL-2, PARP and pro-caspase-9 were also examined. In the miR-1 inhibitor group, the expression levels of PARP were upregulated, while those of BCL-2 and pro-caspase- 9 were decreased compared with the control group (Fig. 4F, H and I). Therefore, these results suggested that the mitochondrial apoptosis pathway was activated following miR-1 inhibition. The increased protein expression levels of cleaved caspase- 3 in the miR-1 inhibitor group compared with the control group was consistent with the increased apoptosis that was observed in the mutant zebrafish compared with that in the WT group. To further analyze the relationship between miR-1 and caspase-3, NCCs were transfected with a miR-1 mimic (Fig. 4L). The results indicated that the expression level of cleaved-caspase-3 was downregulated in cells transfected 
with miR-1 mimics compared with that in the control group (Fig. 4M-O), suggesting that miR-1 inhibited the activation of caspase-3 and affected the apoptosis of NCCs.

Collectively, these results suggested that miR-1 regulated the apoptosis of NCCs via the mitochondrial apoptosis pathway.

\section{Discussion}

In a previous study, a latitudinal expression of miR-1 was observed in WT zebrafish via in situ hybridization, and it was demonstrated that the expression of miR-1 increased during embryonic development at $24 \mathrm{hpf}$ (11).

In the present study, in situ hybridization was used to detect the expression of miR-1 in WT zebrafish embryos. The expression of miR-1 was demonstrated to be primarily localized in the head, pharyngeal region and pectoral fin of zebrafish at $24 \mathrm{hpf}$. Previous findings have indicated that the injection of miR-1 morpholino oligomers in zebrafish resulted in impaired craniofacial chondrogenesis, severe maxillofacial malformations and an abnormal function of NCCs within 4 days post-fertilization. However, it was not clear whether these defects were also affected by the injection of the morpholino (11). Therefore, a miR-1 knockout zebrafish model was generated in the present study to elucidate the role of miR-1 in NC development.

NCCs have been reported to contribute to the development of bones, cartilage, cranial ganglia and connective tissues in the face and neck (1). Previous studies have demonstrated that the abnormal expression of genes associated with $\mathrm{NC}$ may result in craniofacial, bone and cartilage dysplasia due to abnormal cell migration, proliferation and differentiation (26-29). For example, dlx3b has been associated with the differentiation of the pharyngeal arch in zebrafish (30). Also, Ngn1 has been reported to regulate the differentiation of the dorsal root ganglion in zebrafish development (31). In the current study, in situ hybridization evidence collectively suggested that miR-1 serve an important role during the migration and differentiation periods of NCCs.

miRNAs are a class of highly conserved, noncoding RNA molecules with a length of 18-25 nucleotides. The mature sequence of miR-1 is highly conserved among different vertebrates, including zebrafish, mice and humans, which suggests that miR-1 may exhibit similar functions among vertebrates (32). The role of miR-1 in regulating the development of heart, cartilage and liver, among other organs, has been reported (33-35). In the present study, the following abnormal phenotypes were observed in the miR-1-knockout model: Mandibular retraction, shortening of the inferior dental arch and lingual arch, and atypical neural crest-derived tissues, such as abnormal pericardial edema and pigment cells.

Li et al (36) reported that miR-1 decreased cardiomyocyte apoptosis via mediating the expression of apoptosis-related genes in the heart. In the current study, TUNEL staining revealed an increase in apoptosis of pharyngeal NCCs in miR-1 mutant compared with WT zebrafish, which was consistent with previous reports (36). By contrast, $\mathrm{PHH} 3$ immunofluorescence staining did not demonstrate any differences between the WT and mutant groups; additional time points may be required to determine whether alterations in NCC proliferation were caused by miR-1 knockout.
Caspase- 3 has been reported to be a key protein in the mitochondrial apoptosis pathway, and RARP is one of the principal targets of caspase-3 (37). Also, pro-caspase-9 requires to be activated to subsequently cleave and activate caspase-3 (38). Western blotting results suggested that miR-1 may regulate the mitochondrial apoptosis pathway.

The biogenesis of miRNAs is a complex process. After several processing steps (39), mature single-stranded miRNAs have been indicated to enter the miRNA-induced silencing complex, which silences the expression of target genes primarily at the post-transcriptional level (40). The target genes to be silenced are selected via base-pairing interactions between the miRNA and the target mRNA that contains a partial or complete complementary sequence, generally localized in 3'-untranslated region (41). The results of the current study suggested that caspase-3 may be targeted by miR-1. However, the regulatory pathways and mechanisms of this interaction require additional studies.

In conclusion, the present study demonstrated an important role of miR-1 in regulating the apoptosis of NCCs during embryonic development via modulating the mitochondrial apoptosis pathway. A normal expression of miR-1 was identified to be essential for the development of NC derivatives, including the pharyngeal cartilage, mandible and hyoid bone. A thorough understanding of molecular pathway by which miR-1 regulates its target genes may aid the prevention and treatment of miR-1-associated developmental malformations.

\section{Acknowledgements}

Not applicable.

\section{Funding}

The current study was funded by the National Natural Science Foundation of China (grant no. 81771029), the Natural Science Fund for Colleges and Universities in Jiangsu Province, China (grant no. 18KJA320004), the Southeast University-Nanjing Medical University Cooperative Research Project (grant no. JX218GSP20180705) and the Priority Academic Program Development of Jiangsu Higher Education Institutions (grant no. 2014-037).

\section{Availability of data and materials}

All data generated or analyzed during this study are included in this published article.

\section{Authors' contributions}

NZ, WQ, DW, AGR and LY performed the experiments and analyzed the data. NZ, YM, CM, ZX and JM designed the study. NZ, AGR, ZX and JM wrote the manuscript. All authors read and approved the final manuscript.

\section{Ethics approval and consent to participate}

The present study was approved by the Ethics Committee of the School of Stomatology of Nanjing Medical University (approval no. 1403049), and all procedures were performed 
according to the guidelines of the Animal Care Committee of Nanjing Medical University.

\section{Patient consent for publication}

Not applicable.

\section{Competing interests}

The authors declare that they have no competing interests.

\section{References}

1. Mayor R and Theveneau E: The neural crest. Development 140 2247-2251, 2013

2. Trainor PA: Specification and patterning of neural crest cells during craniofacial development. Brain Behav Evol 66: 266-280, 2005.

3. Keyte A and Hutson MR: The neural crest in cardiac congenital anomalies. Differentiation 84: 25-40, 2012.

4. Jones NC, Lynn ML, Gaudenz K, Sakai D, Aoto K, Rey JP, Glynn EF, Ellington L, Du C, Dixon J, et al: Prevention of the neurocristopathy Treacher Collins syndrome through inhibition of p53 function. Nat Med 14: 125-133, 2008.

5. Etchevers HC, Amiel J and Lyonnet S: Molecular bases of human neurocristopathies. Adv Exp Med Biol 589: 213-234, 2006.

6. Chai Y and Maxson RE Jr: Recent advances in craniofacial morphogenesis. Dev Dyn 235: 2353-2375, 2006.

7. Ying SY and Lin SL: Current perspectives in intronic micro RNAs (miRNAs). J Biomed Sci 13: 5-15, 2006.

8. Townley-Tilson WH, Callis TE and Wang D: MicroRNAs 1, 133, and 206: critical factors of skeletal and cardiac muscle development, function, and disease. Int J Biochem Cell Biol 42 $1252-1255,2010$

9. Li P, Wei X, Guan Y, Chen Q, Zhao T, Sun C and Wei L: MicroRNA-1 regulates chondrocyte phenotype by repressing histone deacetylase 4 during growth plate development. FASEB J 28: 3930-3941, 2014.

10. Samal E, Evangelista M, Galang G, Srivastava D, Zhao Y and Vedantham V: Premature microRNA-1 expression causes hypoplasia of the cardiac ventricular conduction system. Front Physiol 10: 235, 2019.

11. Wang D, Weng Y, Guo S, Qin W, Ni J, Yu L, Zhang Y, Zhao Q, Ben J and Ma J: MicroRNA-1 regulates NCC migration and differentiation by targeting sec63. Int J Biol Sci 15: 2538-2547, 2019.

12. Fishman MC: Genomics. Zebrafish - the canonical vertebrate Science 294: 1290-1291, 2001.

13. Liu QY, Wu ZL, Lv WJ, Yan YC and Li YP: Developmental expression of cyclin $\mathrm{H}$ and $\mathrm{Cdk} 7$ in zebrafish: The essential role of cyclin $\mathrm{H}$ during early embryo development. Cell Res 17: 163-173, 2007.

14. Schulte-Merker S, Ho RK, Herrmann BG and Nüsslein-Volhard C: The protein product of the zebrafish homologue of the mouse $\mathrm{T}$ gene is expressed in nuclei of the germ ring and the notochord of the early embryo. Development 116: 1021-1032, 1992

15. de Peralta MS, Mouguelar VS, Sdrigotti MA, Ishiy FA, Fanganiello RD, Passos-Bueno MR, Coux G and Calcaterra NB Cnbp ameliorates Treacher Collins syndrome craniofacial anomalies through a pathway that involves redox-responsive genes. Cell Death Dis 7: e2397, 2016.

16. Ning G, Liu X, Dai M, Meng A and Wang Q: MicroRNA-92a upholds Bmp signaling by targeting noggin 3 during pharyngeal cartilage formation. Dev Cell 24: 283-295, 2013.

17. Babb-Clendenon S, Shen YC, Liu Q, Turner KE, Mills MS, Cook GW, Miller CA, Gattone VH II, Barald KF and Marrs JA: Cadherin-2 participates in the morphogenesis of the zebrafish inner ear. J Cell Sci 119: 5169-5177, 2006.

18. Guo S, Zhang Y, Zhou T, Wang D, Weng Y, Chen Q, Ma J, Li YP and Wang L: GATA4 as a novel regulator involved in the development of the neural crest and craniofacial skeleton via Barx1. Cell Death Differ 25: 1996-2009, 2018

19. Guo S, Zhang Y, Zhou T, Wang D, Weng Y, Wang L and Ma J: Role of GATA binding protein 4 (GATA4) in the regulation of tooth development via GNAI3. Sci Rep 7: 1534, 2017.
20. Lu M, Guo S, Hong F, Zhang Y, Yuan L, Ma C and Ma J: Pax2 is essential for proliferation and osteogenic differentiation of mouse mesenchymal stem cells via Runx2. Exp Cell Res 371: 342-352, 2018

21. Ono W, Sakagami N, Nishimori S, Ono N and Kronenberg HM: Parathyroid hormone receptor signalling in osterix-expressing mesenchymal progenitors is essential for tooth root formation. Nat Commun 7: 11277, 2016.

22. Livak KJ and Schmittgen TD: Analysis of relative gene expression data using real-time quantitative PCR and the 2(-Delta Delta C(T)) Method. Methods 25: 402-408, 2001.

23. Barrallo-Gimeno A, Holzschuh J, Driever W and Knapik EW: Neural crest survival and differentiation in zebrafish depends on mont blanc/tfap2a gene function. Development 131: 1463-1477, 2004.

24. Nelms BL, Pfaltzgraff ER and Labosky PA: Functional interaction between Foxd 3 and Pax 3 in cardiac neural crest development. Genesis 49: 10-23, 2011.

25. Medeiros DM and Crump JG: New perspectives on pharyngeal dorsoventral patterning in development and evolution of the vertebrate jaw. Dev Biol 371: 121-135, 2012.

26. Lake JI, Avetisyan M, Zimmermann AG and Heuckeroth RO: Neural crest requires Impdh2 for development of the enteric nervous system, great vessels, and craniofacial skeleton. Dev Biol 409: 152-165, 2016

27. Lei R, Zhang K, Wei Y, Chen M, Weinstein LS, Hong Y, Zhu M, $\mathrm{Li} \mathrm{H}$ and $\mathrm{Li} \mathrm{H}$ : G-protein $\alpha$-subunit Gs $\alpha$ is required for craniofacial morphogenesis. PLoS One 11: e0147535, 2016.

28. Shao R, Liu J, Yan G, Zhang J, Han Y, Guo J, Xu Z, Yuan Z, Liu J, Malumbres $\mathrm{M}$, et al: $\mathrm{Cdh} 1$ regulates craniofacial development via APC-dependent ubiquitination and activation of Goosecoid. Cell Res 26: 699-712, 2016.

29. Tu CT, Yang TC, Huang HY and Tsai HJ: Zebrafish arl6ip1 is required for neural crest development during embryogenesis. PLoS One 7: e32899, 2012.

30. Alexander C, Piloto S, Le Pabic P and Schilling TF: Wnt signaling interacts with bmp and edn1 to regulate dorsal-ventral patterning and growth of the craniofacial skeleton. PLoS Genet 10: e1004479, 2014

31. Cornell RA and Eisen JS: Delta/Notch signaling promotes formation of zebrafish neural crest by repressing Neurogenin 1 function. Development 129: 2639-2648, 2002.

32. Bartel DP: MicroRNAs: Genomics, biogenesis, mechanism, and function. Cell 116: 281-297, 2004.

33. Fleissner F, Jazbutyte V, Fiedler J, Gupta SK, Yin X, Xu Q, Galuppo P, Kneitz S, Mayr M, Ertl G, et al: Short communication: Asymmetric dimethylarginine impairs angiogenic progenitor cell function in patients with coronary artery disease through a microRNA-21-dependent mechanism. Circ Res 107: 138-143, 2010.

34. Karp X and Ambros V: Developmental biology. Encountering microRNAs in cell fate signaling. Science 310: 1288-1289, 2005.

35. Xu P, Guo M and Hay BA: MicroRNAs and the regulation of cell death. Trends Genet 20: 617-624, 2004.

36. Li W, Liu M,Zhao C, Chen C, Kong Q, Cai Z and Li D: miR-1/133 attenuates cardiomyocyte apoptosis and electrical remodeling in mice with viral myocarditis. Cardiol J 27: 285-294, 2020.

37. Perchellet EM, Wang Y, Weber RL, Sperfslage BJ, Lou K, Crossland J, Hua DH and Perchellet JP: Synthetic 1,4-anthracenedione analogs induce cytochrome c release, caspase-9, -3 , and -8 activities, poly(ADP-ribose) polymerase- 1 cleavage and internucleosomal DNA fragmentation in HL-60 cells by a mechanism which involves caspase-2 activation but not Fas signaling. Biochem Pharmacol 67: 523-537, 2004.

38. Green DR and Reed JC: Mitochondria and apoptosis. Science 281 1309-1312, 1998.

39. Lee Y, Ahn C, Han J, Choi H, Kim J, Yim J, Lee J, Provost P, Rådmark O, Kim S, et al: The nuclear RNase III Drosha initiates microRNA processing. Nature 425: 415-419, 2003.

40. Lin SL and Ying SY: Gene silencing in vitro and in vivo using intronic microRNAs. Methods Mol Biol 1733: 107-126, 2018.

41. Huntzinger E and Izaurralde E: Gene silencing by microRNAs: Contributions of translational repression and mRNA decay. Nat Rev Genet 12: 99-110, 2011.

This work is licensed under a Creative Commons Attribution-NonCommercial-NoDerivatives 4.0 International (CC BY-NC-ND 4.0) License. 